\title{
Review of: "Transcriptome phylogenies support ancient evolutionary transitions in bioluminescence traits of cypridinid ostracods"
}

\author{
Danilo Trabuco do Amaral
}

Potential competing interests: The author(s) declared that no potential competing interests exist.

I'm really glad to read an excellent paper, with a very interesting history, subject, and organisms. Besides, I am impressed with all the carefully methodological details. Appropriate tools and analyses, including comparative pipelines/workflows, were nicely described and used in this study. The bioinformatic approaches used by the authors sound great; however, I would like to know why they used MrBayes instead of Beast software. I know they use the same Bayesian approach, but the second one is exhaustively used for divergence time estimation with more detailed manuals and tutorials available for this purpose.

About the ancestral reconstruction analysis, I'm worried if the lack of some genera in the phylogeny (used 15 of 25 ) would bias the final results/conclusions.

A question that is not so clear to me: The bioluminescence function (defense and courtship) are mutually excluding or the organism may use the light for both purposes. I believe that should be clarified in the text, because that may change the input for the ancestral reconstruction.

The prep-print is well-written and easy to understand and follow. The figures are clean and nice. However, I suggest including in the main text the species name abbreviation. It will be easy to follow it instead to check the supplementary material several times. I also suggested checking once more the grammar to remove any misspelling. The metrics and results themselves are greatly valuable to the scientific community. 